\title{
Initial Experience With Robotic Modified Radical Neck Dissection Using the da Vinci Xi System Through the Bilateral Axillo-Breast Approach
}

\author{
Yun Suk Choi ${ }^{1, *}$ [i] $\cdot$ Yong Tae Hong ${ }^{2, *}$ (i) $\cdot$ Jin Wook Yi ${ }^{1}$ (i) \\ ${ }^{I}$ Department of Surgery, Inha University Hospital, Inha University College of Medicine, Incheon; \\ ${ }^{2}$ Department of Otolaryngology-Head and Neck Surgery, Research Institute for Clinical Medicine of Chonbuk National University \\ and Biomedical Research Institute of Chonbuk National University Hospital, Jeonju, Korea
}

Objectives. The bilateral axillo-breast approach (BABA) to robotic thyroidectomy has been extended to modified radical neck dissection (MRND). This study assessed outcomes in patients who underwent robotic MRND through BABA using the da Vinci Xi system.

Methods. The medical records of 40 patients who underwent total thyroidectomy, bilateral central neck dissection, and MRND from September 2018 to March 2020 were reviewed retrospectively, including 12 who underwent robotic surgery and 28 who underwent open surgery. All operations were performed by a single endocrine surgeon.

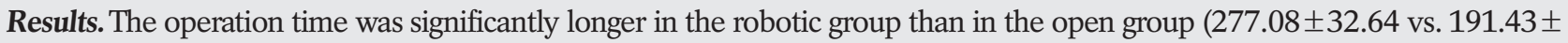
60.43 minutes, respectively, $P<0.01$ ), but the number of retrieved lymph nodes did not differ significantly (32.58 \pm 9.31 vs. $34.54 \pm 10.90$, respectively, $P=0.569$ ). The incidence of transient hypoparathyroidism was significantly lower in the robotic group $(16.7 \%$ [2/12] vs. $53.6 \%$ [15/28], $P=0.041)$. The mean hospital stay was shorter $(3.92 \pm 0.90$ vs. $4.71 \pm 1.63$ days $)$ and the pain score on the first postoperative day was lower $(2.92 \pm 0.29$ vs. $3.18 \pm 0.67)$ in the robotic group. Six of the 12 patients $(50 \%)$ in the robotic group had stimulated thyroglobulin levels $<1.0 \mathrm{ng} / \mathrm{mL}$.

Conclusion. Robotic MRND through BABA has several advantages, including excellent cosmetic outcomes and a lower incidence of transient hypoparathyroidism than is the case for open MRND. Robotic MRND through BABA may be a promising surgical approach compared with conventional open MRND.

Keywords. Thyroid Neoplasms; Neck Dissection; Robotic Surgical Procedures

\section{INTRODUCTION}

Most thyroid cancers are papillary thyroid carcinomas (PTCs), which have been reported to metastasize to cervical lymph nodes (LNs) in $\sim 25 \%-33 \%$ of patients and to lateral LNs in $\sim 5 \%-7 \%$ of patients $[1,2]$. Thus, the treatment of PTC should include re-

- Received July 26, 2020

Revised August 24, 2020

Accepted September 10, 2020

- Corresponding author: Jin WookYi

Department of Surgery, Inha University Hospital, Inha University College

of Medicine, 27 Inhang-ro, Jung-gu, Incheon 22332, Korea

Tel: +82-32-890-3437, Fax: +82-32-890-3549

E-mail: jinwook.yi@inha.ac.kr

*The first two authors contributed equally as co-first authors. moval of both the thyroid gland and metastatic LNs. Modified radical neck dissection (MRND), which includes all compartments from level II to V, is recommended, especially in patients with lateral LN metastasis beyond the border of the carotid artery [3]. Conventionally, thyroid cancer has been treated by transcervical thyroidectomy with $\mathrm{LN}$ dissection. The development of endoscopic and robotic methods has led to the introduction of various types of remote access thyroid surgery.These methods result in minimal neck scarring, which is particularly important as thyroid cancers occur in women more frequently; in the 2017 national cancer statistics in Korea, thyroid cancer occurred in 6,035 men and 20,135 women (https://www.cancer.go.kr). One of these methods, robotic thyroidectomy through the bilateral axillo-breast approach (BABA), using the da Vinci robotic surgical system,

Copyright $\odot 2021$ by Korean Society of Otorhinolaryngology-Head and Neck Surgery.

This is an open-access article distributed under the terms of the Creative Commons Attribution Non-Commercial License (https://creativecommons.org/licenses/by-nc/4.0) which permits unrestricted non-commercial use, distribution, and reproduction in any medium, provided the original work is properly cited. 
was introduced in 2008 [4,5].

Because the working space for thyroidectomy is relatively narrow, and instrument mobilization is restricted, endoscopic thyroidectomy is more difficult to perform than other endoscopic operations. However, endo-wrist actions during robotic operations can facilitate instrument movement compared with an endoscopic operation. Advances in robotic operation systems and surgeons' skills have allowed the performance of more complicated procedures, such as robotic MRND. However, it remains unclear whether robotic MRND is technically and oncologically safe. Robotic MRND can be performed using a retroauricular, transaxillary, or BABA approach [6-12], each of which has several limitations. For example, accessing the contralateral LNs is troublesome using a retroauricular or transaxillary approach, whereas level IV dissection is difficult to perform using BABA [8].

Although several studies have evaluated the safety of robotic MRND through BABA, those studies only evaluated the da Vinci $S$ and da Vinci Si robotic systems $[7,11,12]$. Unlike the da Vinci $\mathrm{S}$ and Si systems, which need a 12-mm trocar for the camera port, the da Vinci Xi system uses all 8-mm trocars, including for the camera port, and provides new endo-wrist instruments such as the vessel sealer extend and suction-irrigation tools. To our knowledge, no study has evaluated the safety of robotic MRND through BABA using the da Vinci Xi system. The present study therefore evaluated the technical and oncologic safety of robotic MRND through BABA using the da Vinci Xi system in comparison with conventional open MRND.

\section{MATERIALS AND METHODS}

The study protocol was approved by the Institutional Review Board of Inha University Hospital (IRB No. INH-2020-07-03). We obtained informed consent about using the photographs of the patients in the article.

\section{Patients and statistics}

From September 2018 to March 2020, 40 patients underwent total thyroidectomy, bilateral central neck dissection, and unilateral MRND for PTC in the Department of Surgery. Of these patients, 12 underwent robotic surgery and 28 underwent open surgery, with all operations performed by a single endocrine sur-

\section{HI I HLIIGHTIS}

- This study is the first to report robotic modified radical neck dissection (MRND) via the bilateral axillo-breast approach using the da Vinci Xi system.

- It provided excellent cosmetic result and oncologic outcomes comparable to those of open surgery. Robotic MRND can be considered as an alternative surgical option. geon. Their medical records and surgical videos were reviewed retrospectively. MRND was indicated for patients with lateral LN metastasis, which was diagnosed by radiologic imaging, fine needle aspiration (FNA), and the washout thyroglobulin ( $\mathrm{Tg}$ ) test. The selection of open or robotic MRND via BABA was dependent mainly on each patent's preference. Robotic MRND via BABA was contraindicated in patients with (1) suspected direct tumor invasion of adjacent structures, including the recurrent laryngeal nerve, trachea, or esophagus (T4 stage); (2) clinically evident metastatic LN with extracapsular spread invading the sternocleidomastoid (SCM) muscle, common carotid artery, or internal jugular vein; (3) metastatic LNs located beneath the level of the clavicle or above the digastric muscle; (4) a history of prior neck surgery or radiation exposure; or (5) a severe comorbidity requiring minimization of operating time.

All patients were permitted to discharge from the 3rd days after surgery, unless otherwise indicated. Vocal cord status was evaluated before and after surgery by laryngeal ultrasound. Permanent vocal cord palsy was defined as non-recovery of vocal cord movement within 6 months. Transient hypoparathyroidism was defined as a parathyroid hormone (PTH) level $<5 \mathrm{pg} / \mathrm{mL}$ within 3 months after surgery. Permanent hypoparathyroidism was defined as serum PTH level $<10 \mathrm{pg} / \mathrm{mL}$ more than 6 months after surgery. The total numbers of retrieved and metastatic LNs, as well as by LN level station (2, 3, 4, 5 and jugular), were recorded. Postoperative pain was evaluated on a visual analog scale (VAS) 1, 2, and 3 days after surgery at 6:00 am on each day, with scores ranging from 0 (no pain) to 10 (extreme pain). All patients were treated postoperatively with radioactive iodine (RAI), as described in the 2015 guidelines of the American Thyroid Association [3], with RAI dose determined by the pathologic aggressiveness of the tumor. Serum concentration of unstimulated $\mathrm{Tg}$ was measured in all patients 3 months after surgery. Thyroid-stimulating hormone (TSH)-stimulated $\mathrm{Tg}$ (sTg) concentration in serum was measured on the day of RAI administration after levothyroxine withdrawal during 3 weeks with dietary iodine restriction, or recombinant TSH injection. The proportion of patients with sTg levels $<1.0 \mathrm{ng} / \mathrm{mL}$ was determined. Because the follow-up periods were relatively short, tumor recurrence was not evaluated.

Continuous variables were compared by unpaired $t$-tests, whereas categorical variables were compared by Fisher's exact tests due to the small numbers of patients. All statistical analyses were performed using IBM SPSS ver. 22.0 (IBM Corp., Armonk, NY, USA).

\section{Surgical procedures for open and robotic MRND}

In open surgery, a long transverse cervical incision was made, followed by total thyroidectomy with central neck node dissection and dissection from level II to V. Robotic total thyroidectomy with MRND using BABA was performed similarly, as described $[7,11,12]$. Patients were placed under general anesthesia, with 

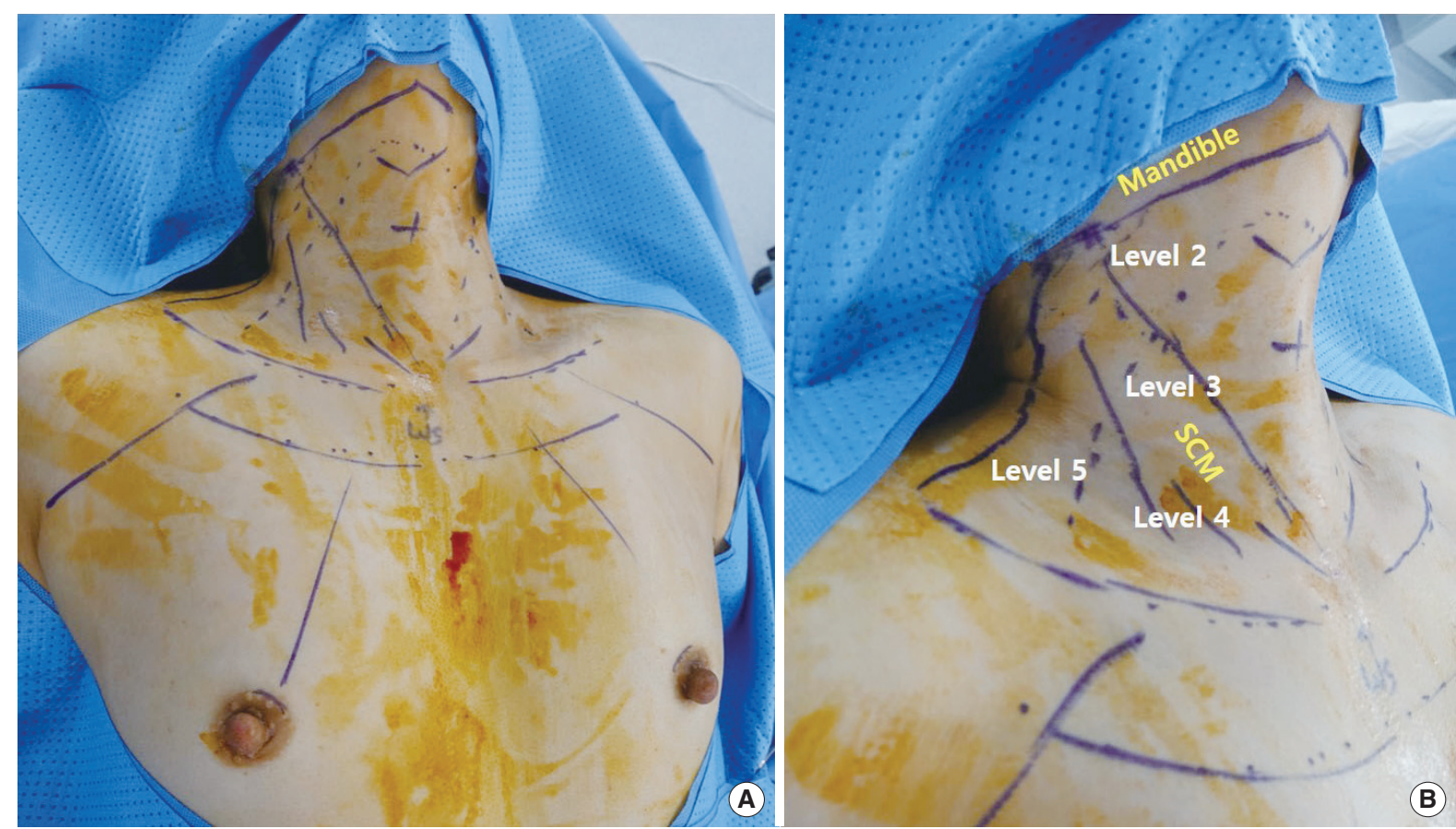

Fig. 1. Flap design for robotic modified radical neck dissection (MRND) via bilateral axillo-breast approach (BABA). (A) Schematic blueprint of robotic BABA flap creation. (B) Extended neck flap for MRND. SCM, sternocleidomastoid.

intraoperative neuromonitoring using a NIM 3.0 device (Medtronic, Minneapolis, MN, USA). All robotic MRNDs via BABA were performed using the da Vinci Xi system (Intuitive Surgical, Sunnyvale, CA, USA). Tissue samples were handled using a Prograsp Forcep and a Long Biopolar Grasper. A permanent cautery hook was used alternately for electrocautery and intraoperative neuromonitoring. Tissue dissection with bleeding control during all robotic MRND procedures was performed using robotic Harmonic ACE (Harmonic) Curved Shears and Vessel Sealer Extend.

Fig. 1 shows the skin markings made for a robotic MRND flap via BABA. Four trocar insertion sites, two in the peri-areolar and two in the axillary creases, each $8 \mathrm{~mm}$ long, were marked for robot arm docking (Fig. 1A). A working space from the incision site to the neck was created by subcutaneous tunneling, with hydrodissection with a 1:200,000 solution of epinephrine in saline. For robotic MRND, the lateral neck flap was dissected further, beyond the posterior border of the SCM muscle and approaching level 5 (Fig. 1B). The superior border of the robotic MRND flap was sited below the mandible, approaching level 2 (Fig. 1B).

After robot docking, thyroidectomy procedures were performed as described [13]. During the MRND phase, the internal jugular vein was exposed by dissection between the strap muscle and the SCM muscle, with the lateral side of the internal jugular vein exposed by cutting the omohyoid muscle (Fig. 2A). Level V LN dissection was performed after separation of the posterior border of the SCM muscle. At that time, Erb's point was checked to identify and preserve the great auricular nerve. During level V
LN dissection, the external jugular vein, spinor accessory nerve, and brachial plexus were preserved (Fig. 2B). After level V LN dissection, the SCM muscle was pulled laterally with surgical silk $\mathrm{U}$ tape through the ipsilateral axillary port to create a working space around the jugular vein and carotid artery (Fig. 2C). Level IV LNs between the SCM muscle and internal jugular vein were dissected, taking care to preserve the transverse cervical artery (Fig. 2D), followed by careful dissection of level III LNs, taking care not to injure the phrenic nerve (Fig. 2E). Level II LNs were subsequently dissected up to the posterior belly of the digastric muscle, taking care not to injure the submandibular gland and vessel injury around the bifurcation of the jugular vein (Fig. 2F). During level II to IV dissection, the jugular vein was carefully mobilized to prevent injury to the vagus nerve, with parts of jugular LNs (LNs attached to the internal jugular vein, especially posterior side of jugular vein) removed. Surgical metal clips are applied on the border of each levels to distinguish node station after specimen removal. Dissected node specimens were placed in a vinyl bag and extracted through the left axillary port. The midline was closed using V-Loc barbed sutures (Medtronic). Two surgical drainage tubes were inserted, one through each axillary port.

\section{RESULTS}

\section{Clinicopathologic characteristics of patients}

The clinicopathologic characteristics of the 40 patients, including 

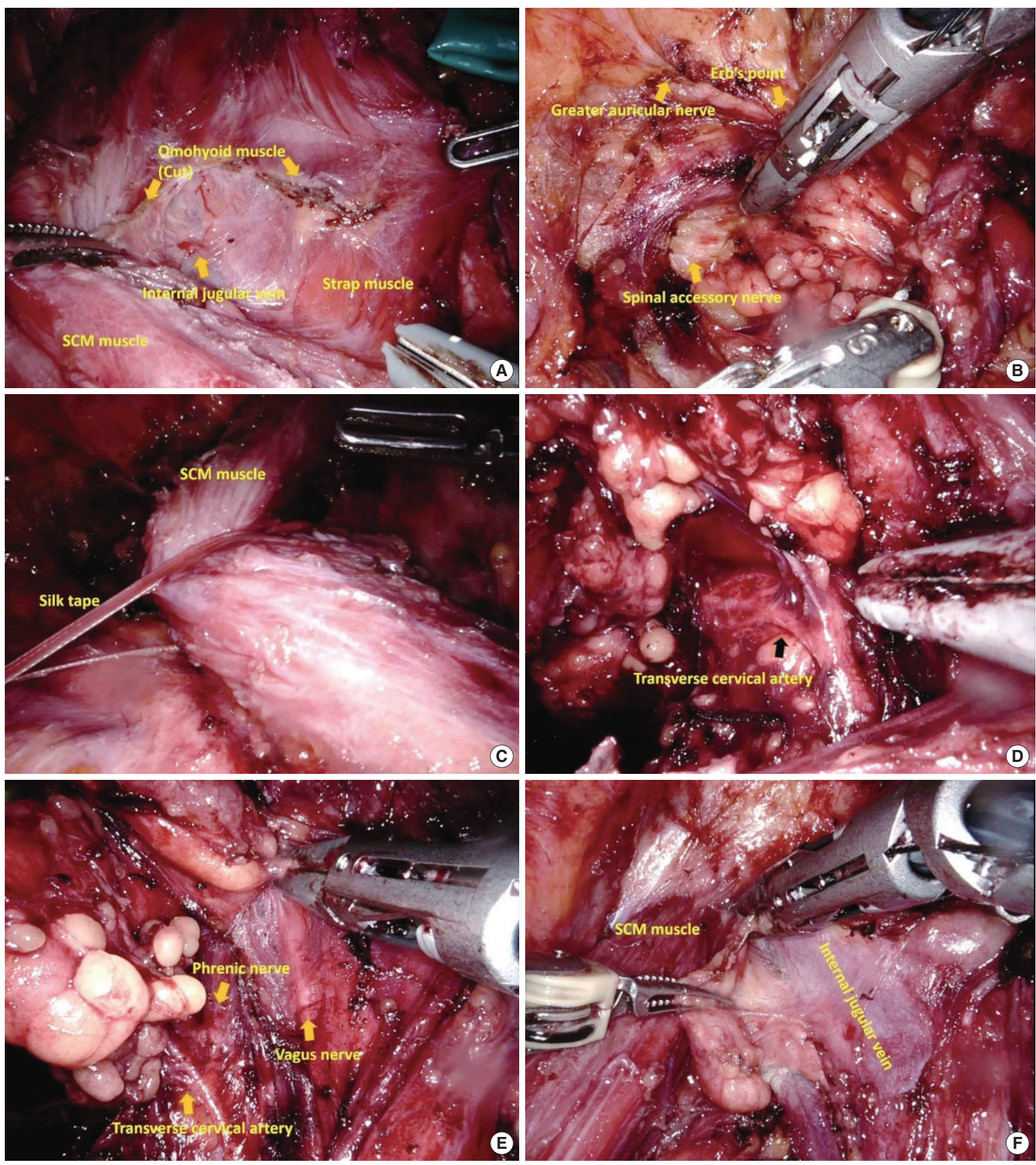

Fig. 2. Procedure for robotic modified radical neck dissection using bilateral axillo-breast approach. (A) Division of the omohyoid muscle to expose the internal jugular vein. (B) Level V lymph node dissection, saving the greater auricular and spinal accessory nerves. (C) Pulling of the sternocleidomastoid (SCM) muscle laterally using silk tape. (D) Level IV node dissection, saving the transverse cervical artery. (E) Level III node dissection, saving the phrenic nerve. (F) Level II node dissection around the internal jugular vein.

12 who underwent robotic and 28 who underwent open MRND, are shown in Table 1 . No patients required conversion from ro- botic to open MRND. There were no significant differences between the two groups in age, sex distribution, location of the 
Table 1. Clinical characteristics of the patients

\begin{tabular}{|c|c|c|c|}
\hline Variable & Open $(n=28)$ & Robotic $(n=12)$ & $P$-value \\
\hline Mean age (yr) & $42.54 \pm 10.15$ & $36.42 \pm 9.91$ & 0.090 \\
\hline Sex & & & 0.716 \\
\hline Male & 10 & 3 & \\
\hline Female & 18 & 9 & \\
\hline Main tumor location & & & 0.612 \\
\hline Right & 11 & 7 & \\
\hline Left & 10 & 3 & \\
\hline Bilateral & 7 & 2 & \\
\hline $\begin{array}{l}\text { Fine needle aspiration } \\
\text { cytology from main tumor }\end{array}$ & & & 0.627 \\
\hline Malignant (category VI) & 25 & 10 & \\
\hline $\begin{array}{l}\text { Suspicious malignant } \\
\text { (category V) }\end{array}$ & 3 & 2 & \\
\hline Neck dissection site & & & 1.000 \\
\hline Left & 13 & 5 & \\
\hline Right & 15 & 7 & \\
\hline Total operation time (min) & $191.43 \pm 60.43$ & $277.08 \pm 32.64$ & $<0.001$ \\
\hline Flap time in robot (min) & & $45.50 \pm 11.90$ & \\
\hline Console time in robot (min) & & $200.33 \pm 26.86$ & \\
\hline Estimated blood loss (mL) & $96.79 \pm 128.06$ & $137.50 \pm 90.77$ & 0.263 \\
\hline
\end{tabular}

Values are presented as mean \pm standard deviation.

primary tumor, MRND site, or the percentage of FNA cytology samples found to be malignant or suspicious for malignancy.The operation time was significantly longer in the robotic than in the open group (277.08 \pm 32.64 vs. $191.43 \pm 60.43$ minutes, respectively; $P<0.001)$. In the robotic group, the mean time for flap creation was $45.50 \pm 11.90$ minutes, and the mean console time was $200.33 \pm 26.86$ minutes. Estimated blood loss was not statistically significantly different between the two groups $(96.79 \pm$ $128.06 \mathrm{~mL}$ in the open group vs. $137.50 \pm 90.77 \mathrm{~mL}$ in the robotic group; $P=0.263$ ).

Table 2 shows the pathologic variables in the open and robotic MRND groups. All 40 patients in both groups were pathologically diagnosed with classic PTC. The mean tumor size did not differ significantly between the robotic and open groups (1.33 \pm 0.72 vs. $1.63 \pm 0.96 \mathrm{~cm}$, respectively; $P=0.294)$. Both microscopic extrathyroidal extension (ETE) and gross ETE were more frequent in the open group, but the differences were not statistically significant; likewise, significant differences were not found for the rates of lymphatic and vascular invasion or $B R A F^{\mathrm{V} 600 \mathrm{E}}$ mutation. The mean number of retrieved LNs $(32.58 \pm 9.31$ vs. $35.54 \pm 10.9, P=0.569$ ) and the mean number of metastatic LNs ( $9.25 \pm 6.87$ vs. $10.71 \pm 6.797, P=0.541)$ did not differ significantly between the robotic and open groups, nor did the number of retrieved LNs from each neck level (Table 2).

\section{Comparison of surgical outcomes}

Table 3 shows the surgical outcomes in both groups. The hospital stay tended to be shorter (3.92 vs. 4.71 days, $P=0.056$ ), and the VAS pain score on the first postoperative day tended to be lower
Table 2. Pathologic variables of the patients

\begin{tabular}{lccc}
\hline Variable & $\begin{array}{c}\text { Open } \\
(\mathrm{n}=28)\end{array}$ & $\begin{array}{c}\text { Robotic } \\
(\mathrm{n}=12)\end{array}$ & $\begin{array}{c}P \text { - } \\
\text { value }\end{array}$ \\
\hline Main tumor size $(\mathrm{cm})$ & $1.63 \pm 0.96$ & $1.33 \pm 0.72$ & 0.294 \\
Extrathyroidal extension & & & \\
$\quad$ Microscopic & 17 & 4 & 0.170 \\
$\quad$ Gross & 9 & 1 & 0.230 \\
Lymphatic invasion & $18(64.3)$ & $11(91.7)$ & 0.124 \\
Vascular invasion & $3(10.7)$ & $1(8.3)$ & 1.000 \\
BRAF'vooE mutation & $22(78.6)$ & $11(91.7)$ & 0.652 \\
Number of retrieved lymph nodes & $34.54 \pm 10.90$ & $32.58 \pm 9.31$ & 0.569 \\
Number of metastatic lymph nodes & $10.71 \pm 6.79$ & $9.25 \pm 6.87$ & 0.541 \\
Number of level II retrieved nodes & $6.96 \pm 5.59$ & $5.17 \pm 1.53$ & 0.125 \\
Number of level III retrieved nodes & $4.89 \pm 2.85$ & $5.33 \pm 1.87$ & 0.568 \\
Number of level IV retrieved nodes & $6.39 \pm 4.99$ & $7.75 \pm 5.38$ & 0.464 \\
Number of level V retrieved nodes & $3.64 \pm 4.26$ & $2.92 \pm 2.23$ & 0.486 \\
Number of bilateral level VI retrieved & $10.61 \pm 5.53$ & $10.33 \pm 5.25$ & 0.883 \\
$\quad$ nodes & & & \\
Number of jugular nodes & $2.07 \pm 2.34$ & $1.08 \pm 1.31$ & 0.100 \\
\hline
\end{tabular}

Values are presented as mean \pm standard deviation or number (\%).

Table 3. Postoperative surgical and oncologic outcomes

\begin{tabular}{lccc}
\hline Variable & $\begin{array}{c}\text { Open } \\
(n=28)\end{array}$ & $\begin{array}{c}\text { Robotic } \\
(n=12)\end{array}$ & $\begin{array}{c}P \text { - } \\
\text { value }\end{array}$ \\
\hline Postoperative hospital stay day & $4.71 \pm 1.63$ & $3.92 \pm 0.90$ & 0.056 \\
VAS on postoperative first day & $3.18 \pm 0.67$ & $2.92 \pm 0.29$ & 0.092 \\
VAS on postoperative second day & $2.68 \pm 0.67$ & $2.58 \pm 0.51$ & 0.630 \\
VAS on postoperative third day & $2.21 \pm 0.74$ & $2.33 \pm 0.78$ & 0.657 \\
PTH on postoperative first day & $8.51 \pm 10.39$ & $10.25 \pm 5.70$ & 0.501 \\
PTH after postoperative 3 months & $23.60 \pm 12.58$ & $31.78 \pm 13.34$ & 0.086 \\
Transient hypoparathyroidism & $15(53.6)$ & $2(16.7)$ & 0.041 \\
Permanent hypoparathyroidism & $3(10.7)$ & 0 & 0.541 \\
Transient vocal cord palsy & $3(10.7)$ & $1(8.3)$ & 1 \\
Permanent vocal cord palsy & $1(3.6)$ & 0 & 1 \\
Tg after postoperative 3 months & $21.31 \pm 103.47$ & $0.29 \pm 0.156$ & 0.292 \\
First radioactive iodine dose & $126.79 \pm 34.65$ & $125.00 \pm 33.71$ & 0.880 \\
TSH level in stimulation & $107.54 \pm 51.38$ & $113.54 \pm 37.51$ & 0.683 \\
TSH-stimulated Tg level (ng/mL) & $35.35 \pm 106.91$ & $1.81 \pm 2.25$ & 0.109 \\
Proportion of stimulated Tg & $10(35.7)$ & $6(50.0)$ & 0.490 \\
$\quad<1.0$ ng/mL & & & \\
\hline
\end{tabular}

Values are presented as mean \pm standard deviation or number (\%). VAS, visual analog scale; PTH, parathyroid hormone; Tg, thyroglobulin; $\mathrm{TSH}$, thyroid-stimulating hormone.

(2.92 vs. $3.18, P=0.092$ ) in the robotic group than in the open group. PTH concentrations measured immediately after the operation and 3 months later did not differ between the two groups. However, the incidence of transient hypoparathyroidism was significantly lower in the robotic group than in the open MRND group (16.7\% [2/12] vs. 53.6\% [15/28], respectively; $P=0.041)$. Permanent hypoparathyroidism did not occur in any patient who underwent robotic MRND. The transient and permanent vocal cord palsy rates did not differ between the two groups.

The mean postoperative serum $\mathrm{Tg}$ concentration tended to be 

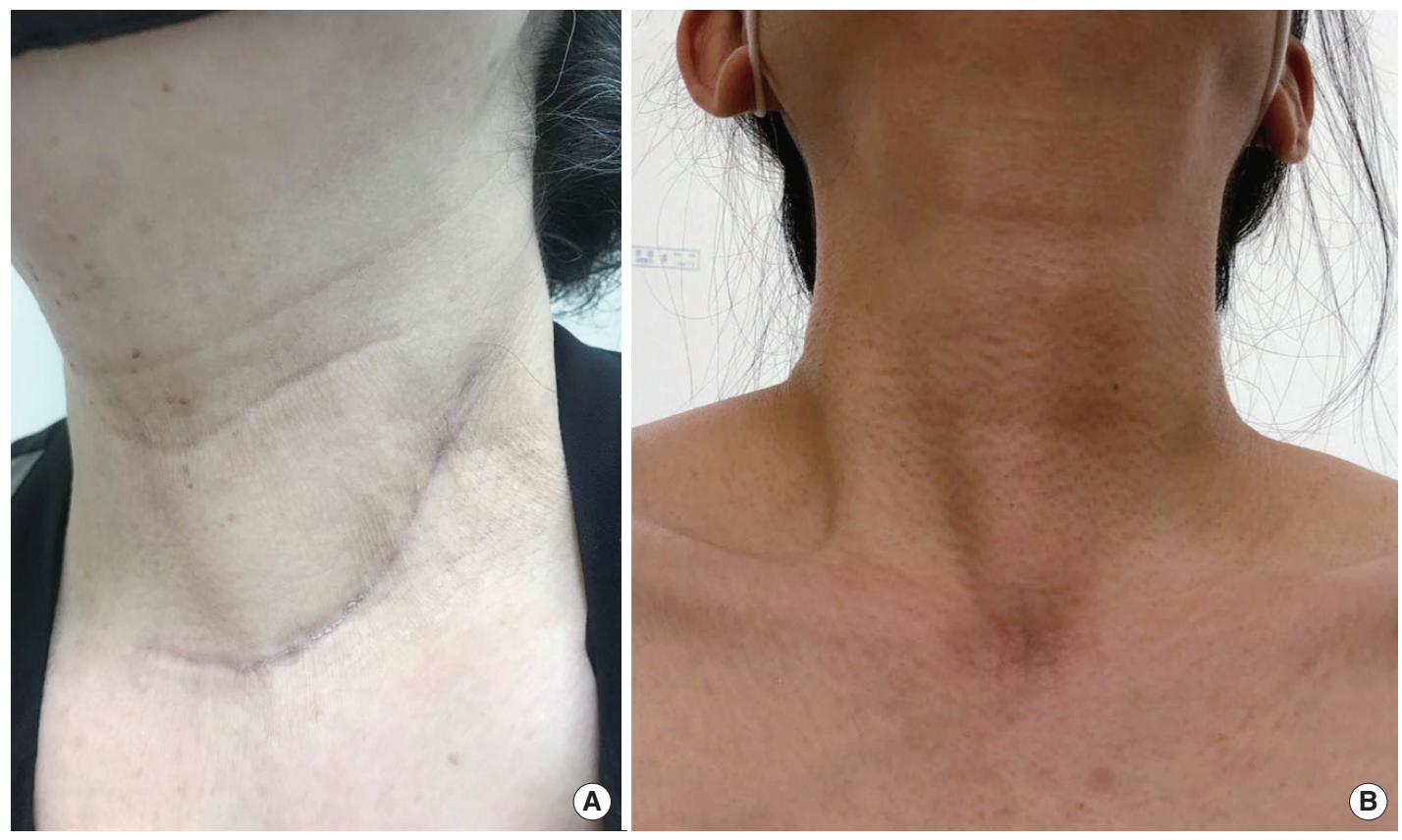

Fig. 3. Comparison of surgical wounds. (A) Scar from open modified radical neck dissection (MRND). (B) No visible neck scar from robotic MRND.

lower in the robotic group than in the open group $(0.29 \pm 0.156$ vs. $21.31 \pm 103.47 \mathrm{ng} / \mathrm{mL}$, respectively; $P=0.292$ ), as did the mean sTg concentration after initial RAI therapy $(1.81 \pm 2.25$ vs. $35.35 \pm 106.91 \mathrm{ng} / \mathrm{mL}$, respectively). The proportion of patients with a sTg level $<1.0 \mathrm{ng} / \mathrm{mL}$ was higher in the robotic group than in the open group $(50.0 \%$ [6/12] vs. $35.7 \%$ [10/28]). A long neck scar (around 10-15 cm) remained in the patients who underwent open MRND, as shown in Fig. 3A. In the robot MRND group, no visible neck scar was present, as shown in Fig. 3B.

\section{DISCUSSION}

Because thyroid cancer mostly affects younger women and has a favorable prognosis, various remote access approaches have been developed to enhance cosmetic outcomes. Neck scars after conventional thyroidectomy are perceived to be less attractive and can reduce patient quality of life $[14,15]$. Patients with metastatic LNs in the lateral neck area require MRND, a more extensive surgical procedure than thyroidectomy [3]. Open MRND requires a 10 - to $15-\mathrm{cm}$-long transverse or hockey stick incision, often leading to an unfavorable thick and long neck scar. Because MRND requires more extensive dissection and is more difficult to perform than thyroidectomy, endoscopic or robotic MRND procedures are performed only at high-volume thyroid centers. Robotic surgical systems have facilitated more difficult procedures, providing a three-dimensional magnified view and enabling the multiarticulated EndoWrist function. Remote access approaches to robotic MRND, including transaxillary, retroauricular, and tran- soral approaches, as well as BABA, may result in scar-less surgery $[6,9,10,15]$. Each of these methods has advantages and limitations in effectively performing MRND. The transaxillary approach provides a view similar to the conventional open approach, as well as a relatively short operation time. However, accessing the upper neck area is relatively difficult, and contralateral MRND is impossible. Moreover, although rare, cases of brachial plexus injuries have been reported [9]. The retroauricular approach can access the upper neck area and requires a smaller area for dissection. However, its operative view is in a direction opposite to that of the open procedure, and contralateral MRND is impossible [8]. Although the transoral approach has been used to dissect lateral neck nodes [15], this approach only allows selective dissection of level III or IV, making it oncologically unsafe at present. Robotic BABA provides a symmetric view, similar to the open approach, and bilateral MRND is possible. However, level IV dissection is relatively difficult because it has an inferior-tosuperior view, and the energy device has limited access to the inferior side of level IV or levelVI $[10,16]$.

To our knowledge, this study is the first to assess robotic MRND via BABA using a new robotic operating system, the da Vinci Xi. The results with this system were comparable to those using the da Vinci S or da Vinci Si system [7,12,17]. Baseline clinical characteristics, including patient age, sex ratio, main tumor location, operation site, and tumor size, did not differ significantly in patients who underwent robotic and open surgery. The operation time was significantly longer in the robotic than in the open group (277.08 \pm 32.64 vs. $191.43 \pm 60.43$ minutes, respectively; $P<0.01$ ). However, when the operation time in the robotic group was sub- 
divided into the flap elevation time (45.50 \pm 11.90 minutes) and console time after robot docking (200.33 \pm 26.86 minutes), the latter did not differ significantly from the time required for open surgery $(P=0.523)$. The rates of postoperative complications, including vocal cord palsy and hypoparathyroidism, were similar in the two groups. One patient in the open surgery group experienced permanent vocal cord palsy due to direct tumor invasion of the recurrent laryngeal nerve.

The incidence of transient hypoparathyroidism was significantly lower in the robotic group ( $16.7 \%$ vs. $53.6 \%, P=0.041)$. Regarding this interesting result, we suggest that following open total thyroidectomy, the naked parathyroid glands are exposed to fresh air during the neck dissection period. These glands may dry up, become cold, or experience oxidative damage during the MRND procedure. In robotic MRND, $\mathrm{CO}_{2}$ gas is insufflated during the entire surgical procedure, keeping the parathyroid glands moist and exposing them to less oxygen, thereby preventing oxidative damage. Moreover, preserving the microvascular structure of the parathyroid glands is easier during robotic surgery. However, these explanations are only hypothetical, and there is no objective evidence supporting them. Therefore, additional studies are required to confirm these hypotheses.

The hospital stay for thyroid surgery is usually longer in South Korea than in western countries because the medical insurance system in South Korea generally covers the cost of longer admissions for cancer patients [18]. Our study found, however, that the mean hospital stay was shorter in the robotic group than in the open group ( $3.92 \pm 0.90$ vs. $4.71 \pm 1.63$ days, respectively; $P=0.056)$. The shorter hospital stay may be linked to the lower postoperative pain score and the significantly lower incidence of transient hypoparathyroidism in the robotic group. The use of BABA alone for remote access thyroidectomy, without neck dissection, has been regarded as not being "minimally invasive" as it requires a wider area of subcutaneous dissection than open surgery $[18,19]$. However, robotic MRND using BABA showed better performance, with excellent cosmetic and clinical outcomes, suggesting that robotic MRND is minimally invasive compared with open MRND.

Regarding oncologic outcomes, the mean number of retrieved LNs and retrieved LNs by neck levels did not differ between the two groups. Use of the da Vinci Xi system for robotic MRND may have several advantages. During robotic MRND using BABA, the visual field is blocked by the clavicle and the camera, making it difficult to access level IV or VI. This limited view of level IV can be overcome by encircling the lower breast using an elastic band, thereby elevating the subareolar height, expanding the visual field of the lower neck compartment [16]. In the da Vinci Xi system, all four robotic arms, including the camera, can be inserted through an 8-mm trocar, allowing the camera and other arms to be changed freely. If another operating view is required, the camera port can be changed not only to another breast site, but to both axillary sites. This can enable dissection of the lower neck compartment, including levels IV and V, and the contralateral side of the neck. Another limitation of robotic MRND was the inability to articulate the ultrasonic shear device (Harmonic), limiting the approach of Harmonic ACE to the lower neck node levels. The da Vinci Xi system, however, allows use of the vessel sealer extend instrument and the bipolar energy coagulator with robot EndoWrist function [17]. The flexible energy device enables safe dissection around all lateral neck node levels. As a result, the number of LNs retrieved in all LN areas did not differ significantly between the two groups $[13,17]$. The number of retrieved LNs in our study was smaller than in previous studies, which may have resulted from differences between pathologists.

A marker of the completeness of thyroid surgery is the sTg level, a reliable indicator of remnant thyroid tissue after total thyroidectomy [19]. In our study, the sTg level and the proportion of patients with sTg levels $<1 \mathrm{ng} / \mathrm{mL}(50.0 \%$ vs. $39.3 \%$, $P=0.530)$ did not differ significantly between the two groups. Other studies measuring sTg concentrations showed the surgical completeness and safety of robotic MRND via BABA [7,11,12].

This study has several limitations, including its single-center and retrospective design, which may have introduced selection bias. In addition, the sample size in both groups was small, and the follow-up period was too short to evaluate recurrence. Because of the limitations of robotic surgery, open surgery is recommended in patients with metastatic LNs invading adjacent structures, including the SCM muscle, trachea, or major vessels. Another disadvantage of robotic surgery is its cost, which is 3-4 times higher than that of open surgery in South Korea $(10,000$ 12,000 vs. $2,500-3,000$ USD). Furthermore, robotic surgery requires additional training, with few hospitals in South Korea currently performing robotic MRND via BABA.

In summary, this study is the first to report the results of robotic MRND via BABA using the da Vinci Xi system. This surgery not only provided excellent cosmetic results, but also surgical and oncologic outcomes comparable to those of open surgery, as well as lower rates of transient hypoparathyroidism. Although longer-term multicenter studies are required, robotic MRND using the da Vinci Xi system via BABA can be considered as an alternative surgical option with excellent cosmetic outcomes.

\section{CONFLICT OF INTEREST}

No potential conflict of interest relevant to this article was reported.

\section{ACKNOWLEDGMENTS}

This work was supported by an Inha University \& Inha University Hospital Research Grant. 


\section{ORCID}

Yun Suk Choi https://orcid.org/0000-0003-2442-664X

Yong Tae Hong https://orcid.org/0000-0001-7584-5823

JinWook Yi https://orcid.org/0000-0002-9296-8443

\section{AUTHOR CONTRIBUTIONS}

Conceptualization \& Data curation: JWY. Formal analysis: YSC. Funding acquisition: JWY. Methodology: YTH, JWY. Project administration \& Visualization: JWY. Writing-original draft: YSC, YTH.Writing-review \& editing: JWY.

\section{REFERENCES}

1. Lee KE, Kim E, Koo do H, Choi JY, Kim KH, Youn YK. Robotic thyroidectomy by bilateral axillo-breast approach: review of 1,026 cases and surgical completeness. Surg Endosc. 2013 Aug;27(8):2955-62.

2. Song J, Yan T, Qiu W, Fan Y, Yang Z. Clinical analysis of risk factors for cervical lymph node metastasis in papillary thyroid microcarcinoma: a retrospective study of 3686 patients. Cancer Manag Res. 2020 Apr;12:2523-30.

3. Haugen BR. 2015 American Thyroid Association management guidelines for adult patients with thyroid nodules and differentiated thyroid cancer: what is new and what has changed? Cancer. 2017 Feb;123(3): 372-81.

4. Lee KE, Choi JY, Youn YK. Bilateral axillo-breast approach robotic thyroidectomy. Surg Laparosc Endosc PercutanTech. 2011 Aug;21(4): 230-6.

5. Lee KE, Koo do H, Kim SJ, Lee J, Park KS, Oh SK, et al. Outcomes of 109 patients with papillary thyroid carcinoma who underwent robotic total thyroidectomy with central node dissection via the bilateral axillo-breast approach. Surgery. 2010 Dec;148(6):1207-13.

6. Byeon HK, Holsinger FC, Tufano RP, Chung HJ, Kim WS, Koh YW, et al. Robotic total thyroidectomy with modified radical neck dissection via unilateral retroauricular approach. Ann Surg Oncol. 2014 Nov;21(12):3872-5.

7. Choi JY, Kang KH. Robotic modified radical neck dissection with bilateral axillo-breast approach. Gland Surg. 2017 Jun;6(3):243-9.
8. Kim K, Kang SW, Chung WY. Robotic-assisted modified radical neck dissection: transaxillary, bilateral axill-breast approach (BABA), Facelift. Curr Surg Rep. 2019;7:20.

9. Kim MJ, Lee J, Lee SG, Choi JB, Kim TH, Ban EJ, et al.Transaxillary robotic modified radical neck dissection: a 5-year assessment of operative and oncologic outcomes. Surg Endosc. 2017 Apr;31(4):1599606.

10. Lee J, Kwon IS, Bae EH, Chung WY. Comparative analysis of oncological outcomes and quality of life after robotic versus conventional open thyroidectomy with modified radical neck dissection in patients with papillary thyroid carcinoma and lateral neck node metastases. J Clin Endocrinol Metab. 2013 Jul;98(7):2701-8.

11. Paek SH, Lee HA, Kwon H, Kang KH, Park SJ. Comparison of robotassisted modified radical neck dissection using a bilateral axillary breast approach with a conventional open procedure after propensity score matching. Surg Endosc. 2020 Feb;34(2):622-7.

12. Yu HW, Chai YJ, Kim SJ, Choi JY, Lee KE. Robotic-assisted modified radical neck dissection using a bilateral axillo-breast approach (robotic BABA MRND) for papillary thyroid carcinoma with lateral lymph node metastasis. Surg Endosc. 2018 May;32(5):2322-7.

13. Yoon HJ, Ahn JH, Kim JH, Yi JW, Hur MH. Initial experience of BABA robotic thyroidectomy using the daVinci Xi system in Incheon, Korea. J Endocr Surg. 2019 Sep;19(3):59-67.

14. Juarez MC, Ishii L, Nellis JC, Bater K, Huynh PP, Fung N, et al. Objectively measuring social attention of thyroid neck scars and transoral surgery using eye tracking. Laryngoscope. 2019 Dec;129(12): 2789-94.

15. Tan Y, Guo B, Deng X, Ding Z,Wu B, Niu Y, et al.Transoral endoscopic selective lateral neck dissection for papillary thyroid carcinoma: a pilot study. Surg Endosc. 2020;34:5274-82.

16. Seup Kim B, Kang KH, Park SJ. Robotic modified radical neck dissection by bilateral axillary breast approach for papillary thyroid carcinoma with lateral neck metastasis. Head Neck. 2015 Jan;37(1): 37-45.

17. Yang SC, Ahn JH, Kim JH, Yi JW, Hur MH, Lee KY. Comparison of the vessel sealer Extend ${ }^{\circledR}$ with harmonic $A C E \AA$ in robotic bilateral axillary-breast approach thyroid surgery. Gland Surg. 2020 Apr;9(2): 164-71.

18. Hong YT, Ahn JH, Kim JH, Yi JW, Hong KH. Bi-institutional experience of transoral endoscopic thyroidectomy: challenges and outcomes. Head Neck. 2020 Aug;42(8):2115-22.

19. Duren M, Siperstein AE, Shen W, Duh QY, Morita E, Clark OH.Value of stimulated serum thyroglobulin levels for detecting persistent or recurrent differentiated thyroid cancer in high- and low-risk patients. Surgery. 1999 Jul;126(1):13-9. 